\title{
Reducible gauge theories in very special relativity
}

\author{
Sudhaker Upadhyay ${ }^{\mathrm{a}}$ \\ Department of Physics, Indian Institute of Technology Kanpur, Kanpur 208016, India
}

Received: 29 September 2015 / Accepted: 27 November 2015 / Published online: 14 December 2015

(C) The Author(s) 2015. This article is published with open access at Springerlink.com

\begin{abstract}
In this paper we analyze the tensor field (reducible gauge) theories in the context of very special relativity (VSR). Particularly, we study the VSR gauge symmetry as well as VSR BRST symmetry of Kalb-Ramond and Abelian 3-form fields involving a fixed null vector. We observe that the Kalb-Ramond and Abelian 3-form fields and corresponding ghosts get masses in the VSR framework. The effective action in VSR-type axial gauge is greatly simplified compared with the VSR-type Lorenz gauge. Further, we quantize these models using a Batalin-Vilkovisy (BV) formulation in VSR.
\end{abstract}

\section{Introduction}

Special relativity (SR) postulates that the laws of physics share many of the symmetries of Maxwell's equations and is valid at the largest energies attainable today [1]. The maximal symmetry group of Maxwell's equations is the conformal group $S U(2,4)$. However, the existence of particles with mass constrains spacetime symmetry to be no greater than the Lorentz group along with spacetime translations (i.e. Poincaré group). The Poincaré group is proposed as the symmetry of nature by SR principles. The possible violation of the Lorentz symmetry has received much attention with new experimental and theoretical challenges. For instance, many theories of quantum gravity predict breaking of some symmetry groups [2,3]. Experiments and astrophysical observations set precise limits upon the parameters illustrating these violations. The spontaneous symmetry breaking of the Lorentz symmetry is assumed in extension of the minimal standard model Refs. [4-7]. On the other hand, the nondynamical tensor fields are introduced to determine the preferred directions that break the Lorentz symmetry. Some of such investigations are discussed by the Myers-Pospelov model [8-10] together with QED in a constant axial vector background [11-13]. The

\footnotetext{
ae-mails: sudhakerupadhyay@gmail.com; sudhaker@iitk.ac.in
}

Lorentz-invariant theories could emerge as effective theories from a more fundamental scheme, which is invariant under VSR groups but not invariant under the full Poincaré group; this is addressed in Ref. [14].

VSR is the set of subgroups of the Poincare group which preserve the constancy of the velocity of light. In this framework, it is proposed that the laws of physics are invariant under the subgroups of Lorentz group $\mathrm{SO}(1,3)$ (with six parameters) rather than the full Lorentz group [14,15]. Two most interesting subgroups of the Lorentz group, the four parameter $S I M(2)$ group, and the three parameter $H O M(2)$ group have the property of rescaling a fixed null vector. The remarkable property of these subgroups of the Lorentz group is that when they are supplemented with $T, P$, or $C P$ the whole Lorentz group will be recovered. VSR has been studied as regards several aspects. For instance, it has been generalized for the inclusion of supersymmetry [16,17]. It admits the generation of a neutrino mass without lepton number and sterile neutrinos violations [15]. Further, it has been discussed in the case of curved spaces $[18,19]$, noncommutativity [20,21], the cosmological constant [22], dark matter [23], cosmology [24], Abelian gauge fields [25], Born-Infeld electrodynamics [26], and non-Abelian gauge fields [27]. Indeed it is not surprising that in spite of a considerable volume of research on VSR, the higher-form gauge theories in VSR are still unstudied. A basic motivation of this paper is to bridge this gap.

Higher-form gauge theories are generalizations of electromagnetism in which the vector potential 1-form is replaced by exterior forms of higher degree. Higher-form gauge theories are important ingredients in supergravity and superstring theory. They are also important for the other branches of physics [28-33]. For instance, the low energy excitations in string theories contain states described by antisymmetric tensor fields [34,35]. The antisymmetric tensor fields help to describe the various supergravity models. The Abelian rank-2 tensor field gets relevance for the classical string theories [36], for the theory of vortex motion in an irrotational, 
incompressible fluid [37,38], for the dual formulation of the Abelian Higgs model $[39,40]$. They are also an ingredient of supergravity multiplets [41] and play a role in anomaly cancellation of certain superstring theories [42]. The 3-form gauge fields are also important for supergravity theories. For instant, $N=1$ supergravity theory in $d=11$ dimensions includes a massless 3 -form gauge field. The study of solutions of such supergravity theory shows that there are two-branes that indeed do provide sources for 3-form fields [43].

In this paper we study the Kalb-Ramond (2-form) and Abelian rank-3 tensor (3-form) gauge theories in VSR. Specifically, we derive the gauge-invariant action for such theories in VSR. We notice that such an action is not invariant under the usual gauge transformation. However, it is invariant under the modified gauge transformation written in terms of the wiggle operator. The equation of motion for a KalbRamond field is derived by which one eventually gets a mass in VSR. A gauge theory cannot be quantized without choosing an appropriate gauge. Therefore, we choose a VSR-type Lorenz gauge to quantize the theory. This gauge is incorporated in the theory by adding a corresponding gauge-fixing term to the gauge-invariant action. To make the theory physically equivalent, the gauge-fixing term induce a ghost term in the path integral. A remarkable feature of such a study is that the ghost fields and ghost of ghost fields also get masses in VSR. Since all these fields acquire a common mass, it cannot be used as an alternative for the Higgs mechanism. Further we compute the BRST symmetry for Kalb-Ramond theory in VSR. To quantize the theory a VSR-type axial-type gauge is also chosen which has a simpler form than the VSR-type Lorenz gauge. We also quantize the theory utilizing the BV formulation where we derive the extended quantum action of the model satisfying the quantum master equation. Further we study an Abelian 3-form gauge theory in VSR. The 3-form gauge field together with various ghost fields also acquires mass in the VSR framework. We further perform the BRST quantization of such a model also in VSR. We also shed light on Abelian 3-form gauge theory in a BV formulation with a similar outcome as in the case of 2-form gauge theory.

This presentation of the paper is as follows. First we discuss the BRST quantization of an Abelian 2-form gauge theory in VSR in Sect. 2. In this section the BV formulation prospects are also studied. In Sect. 3, we analyze Abelian 3 -form gauge theory in VSR. The BRST quantization and BV formulation are also studied in this section. Finally, we conclude the results with remarks on future research in the last section.

\section{Abelian 2-form fields in VSR}

The Maxwell theory is modified in VSR; the same must happen to the Abelian rank-2 tensor (Kalb-Ramond) field theory.
To see this, we start with the field strength tensor in VSR for the Kalb-Ramond tensor field $B_{\mu \nu}$ in VSR involving a fixed null vector $n_{\mu}$ as

$$
\begin{aligned}
F_{\mu \nu \rho}= & \partial_{\mu} B_{\nu \rho}+\partial_{\nu} B_{\rho \mu}+\partial_{\rho} B_{\mu \nu} \\
& +\frac{1}{2} m^{2}\left[n_{\mu} \frac{1}{(n \cdot \partial)^{2}} n^{\alpha}\left(\partial_{\nu} B_{\rho \alpha}+\partial_{\rho} B_{\nu \alpha}\right)\right. \\
& +n_{\nu} \frac{1}{(n \cdot \partial)^{2}} n^{\alpha}\left(\partial_{\rho} B_{\mu \alpha}+\partial_{\mu} B_{\rho \alpha}\right) \\
& \left.+n_{\rho} \frac{1}{(n \cdot \partial)^{2}} n^{\alpha}\left(\partial_{\mu} B_{\nu \alpha}+\partial_{\nu} B_{\mu \alpha}\right)\right] .
\end{aligned}
$$

The null vector $n^{\mu}$ transforms multiplicatively under a VSR transformation so that the terms containing ratios having $n^{\mu}$ are invariant. This field strength tensor is not invariant under the standard gauge transformation $\delta B_{\mu \nu}=\partial_{\mu} \zeta_{\nu}-\partial_{\nu} \zeta_{\mu}$, where $\zeta_{\mu}(x)$ is a vector parameter. Rather, this remains invariant under the following modified (VSR-type) gauge transformation:

$$
\begin{aligned}
\delta B_{\mu \nu} & =\tilde{\partial}_{\mu} \zeta_{\nu}-\tilde{\partial}_{\nu} \zeta_{\mu}, \\
& =\partial_{\mu} \zeta_{\nu}-\partial_{\nu} \zeta_{\mu}-\frac{1}{2} \frac{m^{2}}{n \cdot \partial} n_{\mu} \zeta_{\nu}+\frac{1}{2} \frac{m^{2}}{n \cdot \partial} n_{\nu} \zeta_{\mu},
\end{aligned}
$$

where $\tilde{\partial}_{\mu}=\partial_{\mu}-\frac{1}{2} \frac{m^{2}}{n \cdot \partial} n_{\mu}$ is known as the wiggle operator. To have the usual mass dimension for the wiggle operator, a constant $m$ has to be introduced which fixes the scale of VSR effects.

The gauge-invariant action in VSR describing the massive Kalb-Ramond tensor field is given by

$S_{0}^{(2)}=\frac{1}{12} \int \mathrm{d}^{4} x \tilde{F}_{\mu \nu \rho} \tilde{F}^{\mu \nu \rho}$,

where the wiggle field strength tensor has the following form:

$$
\begin{aligned}
\tilde{F}_{\mu \nu \rho}= & \tilde{\partial}_{\mu} B_{v \rho}+\tilde{\partial}_{\nu} B_{\rho \mu}+\tilde{\partial}_{\rho} B_{\mu \nu}, \\
= & \partial_{\mu} B_{\nu \rho}+\partial_{\nu} B_{\rho \mu}+\partial_{\rho} B_{\mu \nu}-\frac{1}{2} \frac{m^{2}}{n \cdot \partial} n_{\mu} B_{\nu \rho} \\
& -\frac{1}{2} \frac{m^{2}}{n \cdot \partial} n_{\nu} B_{\rho \mu}-\frac{1}{2} \frac{m^{2}}{n \cdot \partial} n_{\rho} B_{\mu \nu} \\
= & F_{\mu \nu \rho}-\frac{1}{2} m^{2}\left(n_{\mu} \frac{1}{(n \cdot \partial)^{2}} n^{\alpha} F_{\nu \rho \alpha}\right. \\
& \left.+n_{\nu} \frac{1}{(n \cdot \partial)^{2}} n^{\alpha} F_{\rho \mu \alpha}+n_{\rho} \frac{1}{(n \cdot \partial)^{2}} n^{\alpha} F_{\mu \nu \alpha}\right) .
\end{aligned}
$$

It is evident from the above relation that $\tilde{F}_{\mu \nu \rho}$ does not coincide with $F_{\mu \nu \rho}$ given in (1).

The equations of motion (EOM) for the Kalb-Ramond field is calculated as

$\tilde{\partial}_{\mu} \tilde{F}^{\mu \nu \rho}=0$.

For the VSR-type Lorenz gauge $\tilde{\partial}_{\mu} B^{\mu v}=0$, the EOM reduces to

$\left[\square-m^{2}\right] B^{v \rho}=0$, 
which remarkably implies that the field $B_{\mu \nu}$ has mass $m$. The non-local terms are dealt with by the following relation [44]:

$\frac{1}{n \cdot \partial}=\frac{1}{\partial_{t}+\partial_{z}}=\int \mathrm{d} t_{+}$

where $t_{+}=\frac{t+z}{2}$. Here we observe that our results are in agreement with [25]. Next we will study the covariant quantization of Abelian 2-form gauge theory in VSR.

\subsection{Different gauges}

In order to quantize a gauge theory we must add a gaugefixing term and the corresponding Faddeev-Popov term to the invariant action. Doing so, the gauge-fixing term breaks the local gauge symmetry and thus the divergence of the functional integral disappears. However, the ghost term improves the integration measure to provide correct predictions for the gauge-invariant observables. Therefore, for the so-called BRST quantization, it is necessary to introduce the following ghost and auxiliary fields for reducible 2-form gauge theory: anticommuting vector fields $\rho_{\mu}$ and $\bar{\rho}_{\mu}$, a commuting vector field $\beta_{\mu}$, anticommuting scalar fields $\chi$ and $\bar{\chi}$, and commuting scalar fields $\sigma, \varphi$, and $\bar{\sigma}$. The gauge fixing and ghost action for an antisymmetric rank-2 tensor field in VSR-type Lorenz gauge is given by

$$
\begin{aligned}
S_{g f+g h}^{(2) L}= & \int \mathrm{d}^{4} x\left[i \bar{\rho}_{\nu} \tilde{\partial}_{\mu}\left(\tilde{\partial}^{\mu} \rho^{\nu}-\tilde{\partial}^{\nu} \rho^{\mu}\right)-\bar{\sigma} \tilde{\partial}_{\mu} \tilde{\partial}^{\mu} \sigma\right. \\
& +\beta_{\nu}\left(\tilde{\partial}_{\mu} B^{\mu \nu}+\lambda_{1} \beta^{\nu}-\partial^{\nu} \varphi\right) \\
& \left.-i \bar{\chi}\left(\tilde{\partial}_{\mu} \rho^{\mu}+\lambda_{2} \chi\right)-i \bar{\rho}^{\mu} \tilde{\partial}_{\mu} \chi\right], \\
= & \int \mathrm{d}^{4} x\left[i \overline { \rho } _ { \nu } \left(\partial_{\mu} \partial^{\mu} \rho^{\nu}-\partial_{\mu} \partial^{\nu} \rho^{\mu}-m^{2} \rho^{\nu}\right.\right. \\
& +\frac{1}{2} \frac{m^{2}}{n \cdot \partial} n^{\nu} \partial \cdot \rho+\frac{1}{2} \frac{m^{2}}{n \cdot \partial} \partial^{\nu} n \cdot \rho \\
& \left.-\frac{1}{4} \frac{m^{2}}{(n \cdot \partial)^{2}} n^{\nu} n \cdot \rho\right)-\bar{\sigma}\left(\partial_{\mu} \partial^{\mu}-m^{2}\right) \sigma \\
& +\beta_{\nu} \partial_{\mu} B^{\mu \nu}-\frac{1}{2} m^{2} \beta_{\nu} \frac{1}{n \cdot \partial} n_{\mu} B^{\mu \nu}+\lambda_{1} \beta_{\nu} \beta^{\nu} \\
& -\beta_{\nu} \partial^{v} \varphi-i \bar{\chi} \partial_{\mu} \rho^{\mu}+\frac{i}{2} m^{2} \bar{\chi} \frac{1}{n \cdot \partial} n_{\mu} \rho^{\mu} \\
& \left.-i \lambda_{2} \bar{\chi} \chi-i \bar{\rho}^{\mu} \partial_{\mu} \chi-\frac{i}{2} \frac{m^{2}}{n \cdot \partial} \bar{\rho}^{\mu} n_{\mu} \chi\right],
\end{aligned}
$$

where $\lambda_{1}$ and $\lambda_{2}$ are gauge parameters. It is evident from the above expression that the ghost fields and ghost of ghost fields have mass $m$ in VSR. Since all the fields acquire a common mass, it cannot be used as a replacement for the Higgs mechanism. The ghost propagator and ghost of ghost propagator are computed, respectively, as

$$
\begin{aligned}
& D_{\mu \nu}^{g h}(k)=-\frac{1}{k^{2}+m^{2}}\left[g_{\mu \nu}+\frac{k_{\mu} k_{v}}{m^{2}}\right], \\
& D^{g g h}(p)=-\frac{1}{p^{2}+m^{2}} .
\end{aligned}
$$

It can be seen that the propagators and vertices have the same large momentum behavior as in Lorentz-invariant theories. So the 2-form gauge theory in VSR is renormalizable.

The expression (8) can further be written in terms of BRST variation $\delta_{b}$ of gauge-fixing fermion $\psi^{L}$ as follows:

$$
\begin{aligned}
S_{g f+g h}^{(2) L}= & \delta_{b} \int \mathrm{d}^{4} x \psi^{L}, \\
= & \delta_{b} \int \mathrm{d}^{4} x\left[-i \bar{\rho}_{\nu}\left(\tilde{\partial}_{\mu} B^{\mu \nu}+\lambda_{1} \beta^{\nu}-\tilde{\partial}^{\nu} \varphi\right)\right. \\
& \left.-i \bar{\sigma}\left(\tilde{\partial}_{\mu} \rho^{\mu}+\lambda_{2} \chi\right)\right], \\
= & \delta_{b} \int \mathrm{d}^{4} x\left[-i \bar{\rho}_{\nu} \partial_{\mu} B^{\mu \nu}+\frac{i}{2} m^{2} \bar{\rho}_{\nu} \frac{1}{n \cdot \partial} n_{\mu} B^{\mu \nu}\right. \\
& -i \lambda_{1} \bar{\rho}_{\nu} \beta^{\nu}+i \bar{\rho}_{\nu} \partial^{\nu} \varphi-\frac{i}{2} m^{2} \bar{\rho}_{\nu} \frac{1}{n \cdot \partial} n^{\nu} \varphi \\
& \left.-i \bar{\sigma}\left(\partial_{\mu} \rho^{\mu}-\frac{1}{2} \frac{m^{2}}{n \cdot \partial} n_{\mu} \rho^{\mu}+\lambda_{2} \chi\right)\right],
\end{aligned}
$$

where the BRST transformation of the fields is given by

$$
\begin{aligned}
\delta_{b} B_{\mu \nu} & =-\left(\partial_{\mu} \rho_{\nu}-\partial_{\nu} \rho_{\mu}-\frac{1}{2} \frac{m^{2}}{n \cdot \partial} n_{\mu} \rho_{\nu}+\frac{1}{2} \frac{m^{2}}{n \cdot \partial} n_{\nu} \rho_{\mu}\right) \Lambda \\
\delta_{b} \rho_{\mu} & =-i\left(\partial_{\mu} \sigma-\frac{1}{2} \frac{m^{2}}{n \cdot \partial} n_{\mu} \sigma\right) \Lambda, \quad \delta_{b} \sigma=0 \\
\delta_{b} \bar{\rho}_{\mu} & =i \beta_{\mu} \Lambda, \quad \delta_{b} \beta_{\mu}=0, \quad \delta_{b} \bar{\sigma}=-\bar{\chi} \Lambda \\
\delta_{b} \varphi & =-\chi \Lambda, \quad \delta_{b} \bar{\chi}=0, \quad \delta_{b} \chi=0 .
\end{aligned}
$$

Here $\Lambda$ is an infinitesimal Grassmann parameter.

The gauge fixing and ghost action for the antisymmetric rank-2 tensor field in VSR-type axial gauge (i.e. $\eta_{\mu} B^{\mu \nu}=0$ ) is given by

$$
\begin{aligned}
S_{g f+g h}^{(2) A}= & \int \mathrm{d}^{4} x\left[i \bar{\rho}_{\nu} \eta_{\mu}\left(\tilde{\partial}^{\mu} \rho^{\nu}-\tilde{\partial}^{v} \rho^{\mu}\right)-\bar{\sigma} \eta_{\mu} \tilde{\partial}^{\mu} \sigma\right. \\
& +\beta_{\nu}\left(\eta_{\mu} B^{\mu \nu}+\lambda_{1} \beta^{\nu}-\eta^{\nu} \varphi\right) \\
& \left.-i \bar{\chi}\left(\eta_{\mu} \rho^{\mu}+\lambda_{2} \chi\right)-i \bar{\rho}^{\mu} \eta_{\mu} \chi\right], \\
= & \int \mathrm{d}^{4} x\left[i \overline { \rho } _ { \nu } \eta _ { \mu } \left(\partial^{\mu} \rho^{\nu}-\partial^{\mu} \rho^{\nu}-\frac{1}{2} \frac{m^{2}}{n \cdot \partial} n^{\mu} \rho^{\nu}\right.\right. \\
& \left.+\frac{1}{2} \frac{m^{2}}{n \cdot \partial} n^{\mu} \rho^{\nu}\right)-\bar{\sigma} \eta_{\mu} \partial^{\mu} \sigma+\frac{1}{2} m^{2} \bar{\sigma} \frac{1}{n \cdot \partial} \eta \cdot n \sigma \\
& +\beta_{\nu}\left(\eta_{\mu} B^{\mu \nu}+\lambda_{1} \beta^{\nu}-\eta^{\nu} \varphi\right) \\
& \left.-i \bar{\chi}\left(\eta_{\mu} \rho^{\mu}+\lambda_{2} \chi\right)-i \bar{\rho}^{\mu} \eta_{\mu} \chi\right] .
\end{aligned}
$$


In terms of a gauge-fixing fermion it can further be written as

$$
\begin{aligned}
S_{g f+g h}^{(2) A}= & \delta_{b} \int \mathrm{d}^{4} x\left[-i \bar{\rho}_{\nu}\left(\eta_{\mu} B^{\mu \nu}+\lambda_{1} \beta^{\nu}-\eta^{\nu} \varphi\right)\right. \\
& \left.-i \bar{\sigma}\left(\eta_{\mu} \rho^{\mu}+\lambda_{2} \chi\right)\right] .
\end{aligned}
$$

We see here that the gauge-fixed action in VSR-type axial gauge has simpler form than the Lorenz gauge. Next we discuss BV formulation of this model.

\subsection{Batalin-Vilkovisky formulation}

To analyze the BV formulation for Abelian rank-2 antisymmetric tensor field theory in VSR, we first define the generating functional in the VSR-type Lorenz gauge in a field/antifield formulation by introducing an antifield corresponding to each field of the theory with opposite statistics, thus:

$$
\begin{aligned}
Z_{2 \text {-form }}^{L}= & \int[\mathrm{d} B \mathrm{~d} \rho \mathrm{d} \bar{\rho} \mathrm{d} \sigma \mathrm{d} \bar{\sigma} \mathrm{d} \varphi \mathrm{d} \chi \mathrm{d} \bar{\chi} \mathrm{d} \beta] \\
& \times \exp \left[i \int \mathrm { d } ^ { 4 } x \left\{\frac{1}{12} F_{\mu \nu \lambda} F^{\mu \nu \lambda}\right.\right. \\
& -B^{\mu \nu \star}\left(\partial_{\mu} \rho_{\nu}-\partial_{\nu} \rho_{\mu}-\frac{1}{2} \frac{m^{2}}{n \cdot \partial} n_{\mu} \rho^{\nu}\right. \\
& \left.+\frac{1}{2} \frac{m^{2}}{n \cdot \partial} n_{\nu} \rho^{\mu}\right)-i \rho^{\mu \star}\left(\partial_{\mu} \sigma-\frac{1}{2} \frac{m^{2}}{n \cdot \partial} n_{\mu} \sigma\right) \\
& \left.\left.+i \bar{\rho}^{\nu \star} \beta_{\nu}-\bar{\sigma}^{\star} \bar{\chi}-\varphi^{\star} \chi\right\}\right] .
\end{aligned}
$$

These antifields (starred fields) are identified with the help of the gauge-fixed fermion given in (10) as

$$
\begin{aligned}
\psi^{L}= & -i \bar{\rho}_{\nu} \partial_{\mu} B^{\mu \nu}+\frac{i}{2} m^{2} \bar{\rho}_{\nu} \frac{1}{n \cdot \partial} n_{\mu} B^{\mu \nu} \\
& -i \lambda_{1} \bar{\rho}_{\nu} \beta^{\nu}+i \bar{\rho}_{\nu} \partial^{\nu} \varphi-\frac{i}{2} m^{2} \bar{\rho}_{\nu} \frac{1}{n \cdot \partial} n^{\nu} \varphi \\
& -i \bar{\sigma}\left(\partial_{\mu} \rho^{\mu}-\frac{1}{2} \frac{m^{2}}{n \cdot \partial} n_{\mu} \rho^{\mu}+\lambda_{2} \chi\right) .
\end{aligned}
$$

These identifications are

$$
\begin{aligned}
B^{\mu \nu \star}= & \frac{\delta \psi^{L}}{\delta B_{\mu \nu}}=i \partial^{\mu} \bar{\rho}^{\nu}+\frac{i}{2} m^{2} \bar{\rho}^{\nu} \frac{1}{n \cdot \partial} n^{\mu}, \\
\bar{\rho}^{\nu \star}= & \frac{\delta \psi^{L}}{\delta \bar{\rho}_{\nu}}=-i\left(\partial_{\mu} B^{\mu \nu}-\frac{1}{2} m^{2} \frac{1}{n \cdot \partial} n_{\mu} B^{\mu \nu}\right. \\
& \left.+\lambda_{1} \beta^{\nu}-\partial^{\nu} \varphi+\frac{1}{2} m^{2} \frac{1}{n \cdot \partial} n^{\nu} \varphi\right), \\
\rho^{\mu \star}= & \frac{\delta \psi^{L}}{\delta \rho_{\mu}}=i \partial^{\mu} \bar{\sigma}+\frac{i}{2} \bar{\sigma} \frac{m^{2}}{n \cdot \partial} n^{\mu}, \\
\bar{\sigma}^{\star}= & \frac{\delta \psi^{L}}{\delta \bar{\sigma}}=-i\left(\partial_{\mu} \rho^{\mu}-\frac{1}{2} \frac{m^{2}}{n \cdot \partial} n_{\mu} \rho^{\mu}+\lambda_{2} \chi\right),
\end{aligned}
$$

$$
\begin{aligned}
\sigma^{\star} & =\frac{\delta \psi^{L}}{\delta \sigma}=0, \quad \chi^{\star}=\frac{\delta \psi^{L}}{\delta \chi}=-i \lambda_{2} \bar{\sigma}, \\
\varphi^{\star} & =\frac{\delta \psi^{L}}{\delta \varphi}=-i \partial_{\mu} \bar{\rho}^{\mu}-\frac{i}{2} m^{2} \bar{\rho}_{\nu} \frac{1}{n \cdot \partial} n^{\nu}, \\
\bar{\chi}^{\star} & =\frac{\delta \psi^{L}}{\delta \bar{\chi}}=0 .
\end{aligned}
$$

This can further be expressed in a compact form as

$$
Z_{2 \text {-form }}^{L}=\int \mathcal{D} \phi \mathrm{e}^{i W_{2 \text {-form }}\left(\phi, \phi^{\star}\right)},
$$

where $W_{2 \text {-form }}\left(\phi, \phi^{\star}\right)$ is an extended quantum action for the Abelian 2-form gauge theory in the VSR-type Lorenz gauge written in terms of generic field $\phi$ and antifield $\phi^{\star}$. It is well known that the value of the generating functional $Z_{2 \text {-form }}^{L}$ does not depend on the choice of the gauge-fixing fermion. This extended quantum action, $W_{2 \text {-form }}\left(\phi, \phi^{\star}\right)$, is the solution of a certain rich mathematical relation, which is called the quantum master equation, given by

$\Delta \mathrm{e}^{i W_{2-\text { form }}\left[\phi, \phi^{\star}\right]}=0, \quad \Delta \equiv(-1)^{\epsilon} \frac{\partial_{l}}{\partial \phi} \frac{\partial_{l}}{\partial \phi^{\star}}$.

Corresponding to different choices of gauge condition, there will be many possible solutions of the quantum master equation. The ghost number and statistics of $\phi^{\star}$ are

$\operatorname{gh}\left[\phi^{\star}\right]=-\operatorname{gh}[\phi]-1, \quad \epsilon\left(\phi^{\star}\right)=\epsilon(\phi)+1(\bmod 2)$.

The quantum action can be extended up to the one-loop order correction as

$W_{2 \text {-form }}\left[\phi, \phi^{\star}\right]=S_{0}[\phi]+S_{g f+g h}^{(2) L}\left[\phi, \phi^{\star}\right]+\hbar M_{1}\left[\phi, \phi^{\star}\right]$,

where $S_{0}+S_{g f+g h}^{(2) L}$ is the complete action given in Eqs. (3) and (10) and $M_{1}$ appears from nontrivial measure factors.

The behavior of $W_{2 \text {-form }}$ for BRST transformations can be given by

$\delta_{b} W_{2 \text {-form }}=i \hbar \Delta W_{2 \text {-form }}$

For (non-anomalous) gauge theory up to first-order correction $M_{1}$ the solution does not depend on antifields. In this situation, the BRST transformations of the complete action $S_{0}+S_{g f+g h}^{(2) L}$ and $M_{1}$ are given by

$\delta_{b}\left(S_{0}+S_{g f+g h}^{(2) L}\right)=0, \quad \delta_{b} M_{1}=i \Delta\left(S_{0}+S_{g f+g h}^{(2) L}\right)$.

This result can further be generalized up to higher order of perturbation.

In the next section we will study the case of an Abelian rank-3 tensor field theory in VSR. 


\section{Abelian 3-form fields in VSR}

The Abelian 3-form gauge field is important for supergravity theory in higher spacetime dimensions. So it is important to study such a gauge field in VSR. Let us start by writing the field strength for the Abelian 3-form gauge theory in arbitrary $d$ dimensions for VSR as

$$
\begin{aligned}
H_{\mu \nu \eta \chi}= & \partial_{\mu} B_{\nu \eta \chi}-\partial_{\nu} B_{\eta \chi \mu}+\partial_{\eta} B_{\chi \mu \nu}-\partial_{\chi} B_{\mu \nu \eta}+\frac{1}{2} m^{2} \\
& \times\left[n_{\mu} \frac{1}{(n \cdot \partial)^{2}} n^{\alpha}\left(\partial_{\nu} B_{\eta \chi \alpha}-\partial_{\eta} B_{\chi \alpha \nu}+\partial_{\chi} B_{\alpha \nu \eta}\right)\right. \\
& -n_{v} \frac{1}{(n \cdot \partial)^{2}} n^{\alpha}\left(\partial_{\eta} B_{\chi \mu \alpha}-\partial_{\chi} B_{\mu \alpha \eta}+\partial_{\mu} B_{\alpha \eta \chi}\right) \\
& +n_{\eta} \frac{1}{(n \cdot \partial)^{2}} n^{\alpha}\left(\partial_{\chi} B_{\mu \nu \alpha}-\partial_{\mu} B_{\nu \alpha \chi}+\partial_{\nu} B_{\alpha \chi \mu}\right) \\
& \left.-n_{\chi} \frac{1}{(n \cdot \partial)^{2}} n^{\alpha}\left(\partial_{\mu} B_{\nu \eta \alpha}-\partial_{\nu} B_{\eta \alpha \mu}+\partial_{\eta} B_{\alpha \mu \nu}\right)\right] .
\end{aligned}
$$

It is straightforward to check that this field strength is not invariant under the standard gauge transformation, $\delta B_{\mu \nu \eta}=$ $\partial_{\mu} \lambda_{\nu \eta}+\partial_{\nu} \lambda_{\eta \mu}+\partial_{\eta} \lambda_{\mu \nu}$. Rather, this is invariant under the following modified (VSR-type) gauge transformation:

$$
\begin{aligned}
\delta B_{\mu \nu \eta}= & \partial_{\mu} \lambda_{\nu \eta}+\partial_{\nu} \lambda_{\eta \mu}+\partial_{\eta} \lambda_{\mu \nu}-\frac{1}{2} \frac{m^{2}}{n \cdot \partial} n_{\mu} \lambda_{\nu \eta} \\
& -\frac{1}{2} \frac{m^{2}}{n \cdot \partial} n_{\nu} \lambda_{\eta \mu}-\frac{1}{2} \frac{m^{2}}{n \cdot \partial} n_{\eta} \lambda_{\mu \nu},
\end{aligned}
$$

where $\lambda_{\mu \nu}$ is a tensor parameter of transformation.

To describe a massive 3-form field we define the VSR-type gauge-invariant action in $d$ dimensions as follows:

$S_{0}=\kappa \int \mathrm{d}^{d} x \tilde{H}_{\mu \nu \eta \chi} \tilde{H}^{\mu \nu \eta \chi}$,

where $\kappa$ is some fixed constant and the wiggle field strength for the 3 -form gauge field is given by

$$
\begin{aligned}
\tilde{H}_{\mu \nu \eta \chi}= & \tilde{\partial}_{\mu} B_{\nu \eta \chi}-\tilde{\partial}_{\nu} B_{\eta \chi \mu}+\tilde{\partial}_{\eta} B_{\chi \mu \nu}-\tilde{\partial}_{\chi} B_{\mu \nu \eta}, \\
= & H_{\mu \nu \eta \chi}+\frac{1}{2} m^{2}\left[n_{\mu} \frac{1}{(n \cdot \partial)^{2}} n^{\alpha} H_{\nu \eta \chi \alpha}\right. \\
& -n_{\nu} \frac{1}{(n \cdot \partial)^{2}} n^{\alpha} H_{\eta \chi \mu \alpha}+n_{\eta} \frac{1}{(n \cdot \partial)^{2}} n^{\alpha} H_{\chi \mu \nu \alpha} \\
& \left.-n_{\chi} \frac{1}{(n \cdot \partial)^{2}} n^{\alpha} H_{\mu \nu \eta \alpha}\right] .
\end{aligned}
$$

From the above expression it is evident that the wiggle field strength does not coincide with the field strength $H_{\mu \nu \eta \chi}$. Now, the EOM for the 3 -form gauge field is calculated by

$\tilde{\partial}_{\mu} \tilde{H}^{\mu \nu \eta \chi}=0$,

which, in turn, for VSR-type Lorenz gauge (i.e. $\tilde{\partial}_{\mu} B^{\mu \nu \eta}$ ) reduces to

$\left(\square-m^{2}\right) B^{\nu \eta \chi}=0$.
This is a Klein-Gordon equation for a massive field. This implies that the 3-form gauge field $B^{\nu \eta \chi}$ has mass $m$.

Since the action (25) respects the VSR-type gauge symmetry, for a perturbative formulation, we need to break the local gauge invariance by adding a gauge-fixing term. When constructing the effective action at higher orders, maintaining unitarity, one has to replace the local gauge symmetry by (global) BRST symmetry. To make the gauge-fixing term BRST invariant, we need to add ghost terms to the effective action. We, therefore, fix a VSR-type Lorenz gauge (i.e. $\tilde{\partial}_{\mu} B^{\mu \nu \eta}=0$ ). Since it is a reducible gauge theory, we need some more fixing for other (ghost) fields. So, this gaugefixing condition is incorporated by adding the following gauge-fixed action together with the induced ghost term:

$$
\begin{aligned}
S_{g f+g h}^{L}= & \int \mathrm{d}^{d} x\left[\tilde{\partial}_{\mu} B^{\mu \nu \eta} B_{\nu \eta}+\frac{1}{2} B_{\mu \nu} \bar{B}^{\mu \nu}\right. \\
& +\left(\tilde{\partial}_{\mu} \bar{c}_{\nu \eta}+\tilde{\partial}_{\nu} \bar{c}_{\eta \mu}+\tilde{\partial}_{\eta} \bar{c}_{\mu \nu}\right) \tilde{\partial}^{\mu} c^{\nu \eta} \\
& -\left(\tilde{\partial}_{\mu} \bar{\beta}_{\nu}-\tilde{\partial}_{\nu} \bar{\beta}_{\mu}\right) \tilde{\partial}^{\mu} \beta^{\nu}-B B_{2} \\
& -\frac{1}{2} B_{1}^{2}+\left(\tilde{\partial}_{\mu} \bar{c}^{\mu \nu}+\tilde{\partial}^{\nu} \bar{c}_{1}\right) f_{v} \\
& -\left(\tilde{\partial}_{\mu} c^{\mu \nu}-\tilde{\partial}^{\nu} c_{1}\right) \bar{F}_{\nu}+\tilde{\partial}_{\mu} \bar{c}_{2} \tilde{\partial}^{\mu} c_{2} \\
& \left.+\tilde{\partial}_{\mu} \beta^{\mu} B_{2}+\tilde{\partial}_{\mu} \phi^{\mu} B_{1}-\tilde{\partial}_{\mu} \bar{\beta}^{\mu} B\right],
\end{aligned}
$$

where antisymmetric ghost and antighost fields $\left(c_{\mu \nu}\right.$ and $\left.\bar{c}_{\mu \nu}\right)$ are Grassmannian and the vector field $\phi_{\mu}$, antisymmetric auxiliary fields $B_{\mu \nu}, \bar{B}_{\mu \nu}$, and auxiliary fields $B, B_{1}, B_{2}$ are bosonic in nature. The ghost of ghosts $\left(\beta_{\mu}\right.$ and $\left.\bar{\beta}_{\mu}\right)$ are bosonic in nature. However, the ghost of ghost of ghosts $\left(c_{2}\right.$ and $\bar{c}_{2}$ ) are fermionic in nature. The rest of the Grassmannian fields $\left(c_{1}, \bar{c}_{1}, f_{\mu}\right.$ and $\left.\bar{F}_{\mu}\right)$ are auxiliary fields. It can easily be seen here that the ghosts $\left(c_{\mu \nu}\right.$ and $\left.\bar{c}_{\mu \nu}\right)$, ghost of ghosts $\left(\beta_{\mu}\right.$ and $\left.\bar{\beta}_{\mu}\right)$, and ghost of ghost of ghosts $\left(c_{2}\right.$ and $\left.\bar{c}_{2}\right)$ have mass $m$.

Expanding the wiggle operation, it reduces to

$$
\begin{aligned}
S_{g f+g h}^{L}= & \int \mathrm{d}^{d} x\left[\partial_{\mu} B^{\mu \nu \eta} B_{\nu \eta}-\frac{1}{2} m^{2} B_{\nu \eta} \frac{1}{n \cdot \partial} n_{\mu} B^{\mu \nu \eta}\right. \\
& +\frac{1}{2} B_{\mu \nu} \bar{B}^{\mu \nu}+\left(\partial_{\mu} \bar{c}_{\nu \eta}+\partial_{\nu} \bar{c}_{\eta \mu}+\partial_{\eta} \bar{c}_{\mu \nu}\right) \partial^{\mu} c^{\nu \eta} \\
& +m^{2} \bar{c}_{\nu \eta} c^{\nu \eta}-m^{2} \partial_{\nu} \bar{c}_{\eta \mu} \frac{1}{n \cdot \partial} n^{\mu} c^{\nu \eta} \\
& -\frac{m^{2}}{n \cdot \partial} n_{\nu} \bar{c}_{\eta \mu} \partial^{\mu} c^{\nu \eta}+\frac{1}{2} \frac{m^{4}}{n \cdot \partial} n_{\nu} \bar{c}_{\eta \mu} \frac{1}{n \cdot \partial} n^{\mu} c^{\nu \eta} \\
& -\left(\partial_{\mu} \bar{\beta}_{\nu}-\partial_{\nu} \bar{\beta}_{\mu}\right) \partial^{\mu} \beta^{\nu}-m^{2} \bar{\beta}_{\nu} \beta^{\nu} \\
& -\frac{1}{2} m^{2} \partial_{\nu} \bar{\beta}_{\mu} \frac{1}{n \cdot \partial} n^{\mu} \beta^{\nu}-\frac{1}{2} \frac{m^{2}}{n \cdot \partial} n_{\nu} \bar{\beta}_{\mu} \partial^{\mu} \beta^{\nu} \\
& +\frac{1}{4} \frac{m^{4}}{n \cdot \partial} n_{\nu} \bar{\beta}_{\mu} \frac{1}{n \cdot \partial} n^{\mu} \beta^{\nu}-B B_{2} \\
& -\frac{1}{2} B_{1}^{2}+\left(\partial_{\mu} \bar{c}^{\mu \nu}\right) f_{\nu}-\frac{1}{2} \frac{m^{2}}{n \cdot \partial} n_{\mu} \bar{c}^{\mu \nu} f_{\nu}
\end{aligned}
$$




$$
\begin{aligned}
& +\partial^{v} \bar{c}_{1} f_{v}-\frac{1}{2} \frac{m^{2}}{n \cdot \partial} n^{\nu} \bar{c}_{1} f_{v}-\left(\partial_{\mu} c^{\mu \nu}\right) \bar{F}_{\nu} \\
& +\frac{1}{2} \frac{m^{2}}{n \cdot \partial} n_{\mu} c^{\mu \nu} \bar{F}_{v}+\partial^{v} c_{1} \bar{F}_{v} \\
& -\frac{1}{2} \frac{m^{2}}{n \cdot \partial} n^{v} c_{1} \bar{F}_{v}-\bar{c}_{2}\left(\partial_{\mu} \partial^{\mu}-m^{2}\right) c_{2} \\
& +\partial_{\mu} \beta^{\mu} B_{2}-\frac{1}{2} \frac{m^{2}}{n \cdot \partial} n_{\mu} \beta^{\mu} B_{2} \\
& +\partial_{\mu} \phi^{\mu} B_{1}-\frac{1}{2} \frac{m^{2}}{n \cdot \partial} n_{\mu} \phi^{\mu} B_{1}-\partial_{\mu} \bar{\beta}^{\mu} B \\
& \left.+\frac{1}{2} \frac{m^{2}}{n \cdot \partial} n_{\mu} \bar{\beta}^{\mu} B\right] .
\end{aligned}
$$

The effective action together with (25) and (30) remains invariant under the following set of BRST transformations:

$$
\begin{aligned}
\delta_{b} B_{\mu \nu \eta}= & -\left(\partial_{\mu} c_{\nu \eta}+\partial_{\nu} c_{\eta \mu}+\partial_{\eta} c_{\mu \nu}-\frac{1}{2} \frac{m^{2}}{n \cdot \partial} n_{\mu} c_{\nu \eta}\right. \\
& \left.-\frac{1}{2} \frac{m^{2}}{n \cdot \partial} n_{\nu} c_{\eta \mu}-\frac{1}{2} \frac{m^{2}}{n \cdot \partial} n_{\eta} c_{\mu \nu}\right) \Lambda \\
\delta_{b} c_{\mu \nu}= & \left(\partial_{\mu} \beta_{\nu}-\partial_{\nu} \beta_{\mu}-\frac{1}{2} \frac{m^{2}}{n \cdot \partial} n_{\mu} \beta_{\nu}+\frac{1}{2} \frac{m^{2}}{n \cdot \partial} n_{\nu} \beta_{\mu}\right) \Lambda, \\
\delta_{b} \bar{c}_{\mu \nu}= & B_{\mu \nu} \Lambda, \\
\delta_{b} \bar{B}_{\mu \nu}= & -\left(\partial_{\mu} f_{\nu}-\partial_{\nu} f_{\mu}-\frac{1}{2} \frac{m^{2}}{n \cdot \partial} n_{\mu} f_{\nu}+\frac{1}{2} \frac{m^{2}}{n \cdot \partial} n_{\nu} f_{\mu}\right) \Lambda, \\
\delta_{b} \bar{\beta}_{\mu}= & -\bar{F}_{\mu} \Lambda, \\
\delta_{b} \beta_{\mu}= & -\left(\partial_{\mu} c_{2}-\frac{1}{2} \frac{m^{2}}{n \cdot \partial} n_{\mu} c_{2}\right) \Lambda, \quad \delta_{b} \bar{c}_{2}=B_{2} \Lambda, \\
\delta_{b} c_{1}= & -B \Lambda, \\
\delta_{b} \phi_{\mu}= & -f_{\mu} \Lambda, \quad \delta_{b} \bar{c}_{1}=B_{1} \Lambda, \\
\delta_{b} \mathcal{M}= & 0, \quad \mathcal{M} \equiv\left\{c_{2}, f_{\mu}, \bar{F}_{\mu}, B, B_{1}, B_{2}, B_{\mu \nu}\right\}
\end{aligned}
$$

where $\Lambda$ is the fermionic transformation parameter. The gauge-fixing fermion is given by

$$
\begin{aligned}
\psi_{L}= & -\partial_{\mu} \bar{c}_{\nu \eta} B^{\mu \nu \eta}+\frac{1}{2} \frac{m^{2}}{n \cdot \partial} n_{\mu} \bar{c}_{\nu \eta} B^{\mu \nu \eta}-\frac{1}{2} \bar{c}_{2} B \\
& +\frac{1}{2} c_{1} B_{2}-\frac{1}{2} \bar{c}_{1} B_{1}-c^{\mu \nu} \partial_{\mu} \bar{\beta}_{\nu}+c_{1} \partial_{\mu} \bar{\beta}^{\mu} \\
& +\frac{1}{2} c^{\mu \nu} \frac{m^{2}}{n \cdot \partial} n_{\mu} \bar{\beta}_{\nu}-\partial_{\mu} \bar{c}_{2} \beta^{\mu}+\frac{1}{2} \frac{m^{2}}{n \cdot \partial} n_{\mu} \bar{c}_{2} \beta^{\mu} \\
& +\frac{1}{2} \bar{c}_{\mu \nu} \bar{B}^{\mu \nu}+\bar{c}_{1} \partial_{\mu} \phi^{\mu}-\frac{1}{2} \bar{c}_{1} \frac{m^{2}}{n \cdot \partial} n_{\mu} \phi^{\mu} .
\end{aligned}
$$

This expression will play an important role in the next subsection to get an identification for the antifields in VSR-type Lorenz gauge.

\subsection{Batalin-Vilkovisky formulation}

To describe 3-form gauge theory in BV formulation in VSR, we introduce the antifields corresponding to each field of the model with opposite statistics having non-vanishing BRST symmetry in the generating functional as follows:

$$
\begin{aligned}
Z_{3 \text {-form }}^{L}= & \int \mathcal{D} \phi \exp \left[i \int \mathrm { d } ^ { d } x \left\{\frac{1}{24} F_{\mu \nu \eta \chi} F^{\mu \nu \eta \chi}-B_{\mu \nu \eta}^{\star}\right.\right. \\
& \times\left(\partial^{\mu} c^{\nu \eta}+\partial^{\nu} c^{\eta \mu}+\partial^{\eta} c^{\mu \nu}-\frac{1}{2} \frac{m^{2}}{n \cdot \partial} n_{\mu} c_{\nu \eta}\right. \\
& \left.-\frac{1}{2} \frac{m^{2}}{n \cdot \partial} n_{\nu} c_{\eta \mu}-\frac{1}{2} \frac{m^{2}}{n \cdot \partial} n_{\eta} c_{\mu \nu}\right) \\
& +c_{\mu \nu}^{\star}\left(\partial^{\mu} \beta^{\nu}-\partial^{v} \beta^{\mu}-\frac{1}{2} \frac{m^{2}}{n \cdot \partial} n_{\mu} \beta_{v}\right. \\
& \left.+\frac{1}{2} \frac{m^{2}}{n \cdot \partial} n_{\nu} \beta_{\mu}\right)+\bar{c}_{\mu \nu}^{\star} B^{\mu \nu} \\
& -\bar{B}_{\mu \nu}^{\star}\left(\partial^{\mu} f^{\nu}-\partial^{\nu} f^{\mu}-\frac{1}{2} \frac{m^{2}}{n \cdot \partial} n_{\mu} f_{\nu}+\frac{1}{2} \frac{m^{2}}{n \cdot \partial} n_{v} f_{\mu}\right) \\
& -\beta_{\mu}^{\star}\left(\partial^{\mu} c_{2}-\frac{1}{2} \frac{m^{2}}{n \cdot \partial} n_{\mu} c_{2}\right)-\bar{\beta}_{\mu}^{\star} \bar{F}^{\mu} \\
& \left.\left.+\bar{c}_{2}^{\star} B_{2}+\bar{c}_{1}^{\star} B_{1}-c_{1}^{\star} B-\phi_{\mu}^{\star} f^{\mu}\right\}\right] .
\end{aligned}
$$

These antifields (starred fields) are evaluated with the help of the gauge-fixing fermion (32) as follows:

$$
\begin{aligned}
& B_{\mu \nu \eta}^{\star}=-\partial_{\mu} \bar{c}_{\nu \eta}+\frac{1}{2} \frac{m^{2}}{n \cdot \partial} n_{\mu} \bar{c}_{\nu \eta} \\
& c_{\mu \nu}^{\star}=-\partial_{\mu} \bar{\beta}_{\nu}+\frac{1}{2} \frac{m^{2}}{n \cdot \partial} n_{\mu} \bar{\beta}_{\nu}, \quad \bar{B}_{\mu \nu}^{\star}=\frac{1}{2} \bar{c}_{\mu \nu}, \\
& \bar{c}_{\mu \nu}^{\star}=\frac{1}{2} \bar{B}_{\mu \nu}+\partial^{\eta} B_{\mu \nu \eta}-\frac{1}{2} \frac{m^{2}}{n \cdot \partial} n^{\eta} B_{\eta \mu \nu}, \\
& \beta_{\mu}^{\star}=-\partial_{\mu} \bar{c}_{2}+\frac{1}{2} \frac{m^{2}}{n \cdot \partial} n_{\mu} \bar{c}_{2}, \\
& \bar{\beta}_{\mu}^{\star}=-\partial_{\mu} c_{1}+\partial^{\nu} c_{\nu \mu}-\frac{1}{2} c_{\nu \mu} \frac{m^{2}}{n \cdot \partial} n^{\nu}, \\
& \bar{c}_{2}^{\star}=-\frac{1}{2} B+\partial_{\mu} \beta^{\mu}-\frac{1}{2} \frac{m^{2}}{n \cdot \partial} n_{\mu} \beta^{\mu}, \\
& c_{1}^{\star}=\frac{1}{2} B_{2}+\partial_{\mu} \bar{\beta}^{\mu}, \\
& \bar{c}_{1}^{\star}=-\frac{1}{2} B_{1}+\partial_{\mu} \phi^{\mu}-\frac{1}{2} \bar{c}_{1} \frac{m^{2}}{n \cdot \partial} n_{\mu} \phi^{\mu}, \\
& \phi_{\mu}^{\star}=-\partial_{\mu} \bar{c}_{1}-\frac{1}{2} \frac{m^{2}}{n \cdot \partial} n_{\mu}, \\
& B^{\star}=-\frac{1}{2} \bar{c}_{2}, \quad B_{1}^{\star}=-\frac{1}{2} \bar{c}_{1}, \quad\left\{B_{\mu \nu}^{\star}, \bar{F}_{\mu}^{\star}, f_{\mu}^{\star}, c_{2}^{\star}\right\}=0 .
\end{aligned}
$$

The generating functional can further be written in compact form as

$Z_{3 \text {-form }}^{L}=\int \mathcal{D} \phi \mathrm{e}^{i W_{3-\text { form }}\left(\phi, \phi^{\star}\right)}$,

where $W_{3 \text {-form }}\left(\phi, \phi^{\star}\right)$ is an extended quantum action for Abelian 3-form gauge theory in the VSR-type Lorenz gauge written in terms of generic field $\phi$ and antifield $\phi^{\star}$. This 
extended quantum action, $W_{3 \text {-form }}\left(\phi, \phi^{\star}\right)$, is the solution of a certain rich mathematical relation, which is called the quantum master equation, given by

$\Delta e^{i W_{3-\text { form }}\left[\phi, \phi^{\star}\right]}=0, \quad \Delta \equiv(-1)^{\epsilon} \frac{\partial_{l}}{\partial \phi} \frac{\partial_{l}}{\partial \phi^{\star}}$.

Here $W_{3 \text {-form }}\left[\phi, \phi^{\star}\right]$ is the solution of the quantum master equation. For different gauge choices, it corresponds to different solutions of the quantum master equation. From this quantum master equation one can get a relation between different correlation functions.

\section{Conclusion}

In this paper we have analyzed the reducible gauge theories in VSR. To be more specific, we have demonstrated the Kalb-Ramond field theory in VSR involving a fixed null vector. We have derived the classical action for such a theory in VSR. We have found that the action is not invariant under the standard gauge transformation for the Kalb-Ramond field. However, such an action remains invariant under the modified (VSR-type) gauge transformation written in terms of the wiggle operator. We have derived the equations of motion for a Kalb-Ramond field, which eventually turns out to be the Klein-Gordon equation for a massive field. This ensures that the Kalb-Ramond field in VSR gets a mass. Further, to quantize such a theory in VSR we have fixed a VSR-type Lorenz gauge which breaks the local (VSR) gauge symmetry. The propagators have also been calculated. This gauge-fixing term induced a ghost term in the path integral. Here we have observed that the ghost fields and ghost of ghost fields also get a common mass in VSR. Therefore it cannot be an alternative to the Higgs mechanism. Further we demonstrate the BRST symmetry for Kalb-Ramond gauge theory in VSR. To break the gauge symmetry an axial-type gauge has also been chosen which has a simpler form than the Lorenz type gauge. We have also quantized the theory utilizing a BV formulation where we derive the extended quantum action of the model to the first order in perturbation satisfying the quantum master equation.

Subsequently, we have considered an Abelian 3-form gauge theory (another reducible gauge theory) also in VSR which plays an important role in 11 dimensional supergravity. It has been shown that this model also respects a VSRtype gauge invariance rather than standard gauge symmetry. We have found that the gauge fields together with all ghost fields get a common mass for such a theory in VSR. We have also analyzed the BRST quantization of 3-form gauge theory in VSR. Further, we have studied the model in the BV formulation. Now it would be extremely interesting to evaluate the different identities following the BRST symmetry of the reducible gauge theories in VSR. This might be helpful in gaining a clear understanding of the theory in VSR. It will also be interesting to explore such results in further interesting models such as perturbative quantum gravity, superYang-Mills theory, supersymmetric Chern-Simons theory, etc.

Open Access This article is distributed under the terms of the Creative Commons Attribution 4.0 International License (http://creativecomm ons.org/licenses/by/4.0/), which permits unrestricted use, distribution, and reproduction in any medium, provided you give appropriate credit to the original author(s) and the source, provide a link to the Creative Commons license, and indicate if changes were made.

Funded by SCOAP $^{3}$.

\section{References}

1. Pierre Auger Collaboration, Phys. Rev. Lett. 101, 061101 (2008)

2. J. Alfaro, H. Morales-Tecotl, L.F. Urrutia, Phys. Rev. Lett. 84, 2318 (2000)

3. J. Alfaro, H. Morales-Tecotl, L.F. Urrutia, Phys. Rev. D 65, 103509 (2002)

4. D. Colladay, V.A. Kostelecky, Phys. Rev. D 55, 6760 (1997)

5. D. Colladay, V.A. Kostelecky, Phys. Rev. D 58, 116002 (1998)

6. For a review, see, for example, in Proceedings of the Meeting on CPT and Lorentz Symmetry, ed. by V.A. Kostelecky (World Scientific, Singapore, 1999)

7. For a review, see, for example, in Proceedings of the Second, Third and Fourth Meeting on CPT and Lorentz. Symmetry, ed. by V.A. Kostelecky (World Scientific, Singapore, 1999)

8. R.C. Myers, M. Pospelov, Phys. Rev. Lett. 90, 211601 (2003)

9. C.M. Reyes, L.F. Urrutia, J.D. Vergara, Phys. Rev. D 78, 125011 (2008)

10. C.M. Reyes, L.F. Urrutia, J.D. Vergara, Phys. Lett. B 675, 336 (2009)

11. A.A. Andrianov, P. Giacconi, R. Soldati, J. High Energy Phys. 02, 030 (2002)

12. J. Alfaro, A.A. Andrianov, M. Cambiaso, P. Giacconi, R. Soldati, Phys. Lett. B 639, 586 (2006)

13. J. Alfaro, A.A. Andrianov, M. Cambiaso, P. Giacconi, R. Soldati, Int. J. Mod. Phys. A 25, 3271 (2010)

14. A.G. Cohen, S.L. Glashow, Phys. Rev. Lett. 97, 021601 (2006)

15. A.G. Cohen, S.L. Glashow, arXiv:hep-ph/0605036

16. A.G. Cohen, D.Z. Freedman, J. High Energy Phys. 0707, 039 (2007)

17. J. Vohanka, Phys. Rev. D 85, 105009 (2012)

18. G. Gibbons, J. Gomis, C. Pope, General very special relativity is Finsler geometry. Phys. Rev. D 76, 081701 (2007)

19. W. Muck, Phys. Lett. B 670, 95 (2008)

20. M.M. Sheikh-Jabbari, A. Tureanu, Phys. Rev. Lett. 101, 261601 (2008)

21. S. Das, S. Ghosh, S. Mignemi, Phys. Lett. A 375, 3237 (2011)

22. E. Alvarez, R. Vidal, Phys. Rev. D 77, 127702 (2008)

23. D.V. Ahluwalia, S.P. Horvath, J. High Energy Phys. 11, 078 (2010)

24. Z. Chang, M.-H. Li, X. Li, S. Wang, Eur. Phys. J. C 73, 2459 (2013)

25. S. Cheon, C. Lee, S. Lee, Phys. Lett. B 679, 73 (2009)

26. R. Bufalo, Phys. Lett. B 746, 251 (2015)

27. J. Alfaro, V.O. Rivelles, Phys. Lett. B 734, 239 (2014)

28. M. Green, J. Schwarz, E. Witten, Superstring Theory (Cambridge University Press, Cambridge, 1987)

29. J. Polchinski, String Theory (Cambridge University Press, Cambridge, 1998) 
30. S. Upadhyay, EPL 103, 61002 (2013)

31. S. Upadhyay, M.K. Dwivedi, B.P. Mandal, Int. J. Mod. Phys. A 28, 1350033 (2013)

32. S. Upadhyay, B.P. Mandal, Eur. Phys. J. C 72, 2059 (2012)

33. S. Upadhyay, B.P. Mandal, Mod. Phys. Lett. A 25, 3347 (2010)

34. M.B. Green, J.H. Schwarz, E. Witten, Superstring Theory (Cambridge University Press, Cambridge, 1987)

35. J. Polchinski, String Theory (Cambridge University Press, Cambridge, 1998)

36. M. Kalb, P. Ramond, Phys. Rev. D 9, 2273 (1974)
37. F. Lund, T. Regge, Phys. Rev. D 14, 1524 (1976)

38. M. Sato, S. Yahikozawa, Nucl. Phys. B 436, 100 (1995)

39. A. Sugamoto, Phys. Rev. D 19, 1820 (1979)

40. R.L. Davis, E.P.S. Shellard, Phys. Lett. B 214, 219 (1988)

41. A. Salam, E. Sezgin, Supergravities in Diverse Dimensions (NorthHolland/World Scientific, Amsterdam/Singapore, 1989)

42. S. Deguchi, T. Mukai, T. Nakajima, Phys. Rev. D 59, 065003 (1999)

43. M.J. Duff, K. Stelle, Phys. Lett. B 253, 113 (1991)

44. A.C. Nayak, R.K. Verma, P. Jain, JCAP 07, 031 (2015) 